\title{
Overview of the III International Workshop on Modeling, Information Processing and Computing (MIP: Computing-2021)
}

\author{
A.A. Voroshilova ${ }^{1,2,3, *}$, A.S. Kuznetsov ${ }^{1}$ \\ ${ }^{1}$ Siberian Federal University, Krasnoyarsk, Russia \\ ${ }^{2}$ Krasnoyarsk Science and Technology City Hall of the Russian Union of Scientific and \\ Engineering Associations, Krasnoyarsk, Russia \\ ${ }^{3}$ Siberian Scientific Centre DNIT, Krasnoyarsk, Russia \\ *E-mail: krasnio@bk.ru
}

\begin{abstract}
The article provides an overview of the main directions of the scientific program of the III International Workshop on Modeling, Information Processing and Computing - MIP: Computing-2021, which took place on May 28, 2021 in Krasnoyarsk on the basis of the ${ }^{2}$ Krasnoyarsk Science and Technology City Hall of the Russian Union of Scientific and Engineering Associations. Provides overview information about the reports that were included in the program of the scientific session of the seminar and presented in the form of online / video reports and presentations of the participants with placement on the website of the conference materials. Information is given about the seminar partner - the service of scientific publications with open access CEUR-WS.org on Sun SITE Central Europe, operating under the auspices of the Technical University in Aachen, Germany (RWTH Aachen University). Proceedings of the MIP: Computing 2021 Seminar are published as articles in English in CEUR Workshop Proceedings, Volume 2899.
\end{abstract}

Keywords: information technology, computer modeling, informatics, computer technology 


\title{
Обзор III Международного семинара MIP: Computing-2021: Компьютерное моделирование, информационные и вычислительные технологии
}

\author{
А.А. Ворошилова ${ }^{1,2,3, *}$, А.С. Кузнецов ${ }^{1}$ \\ ${ }^{1}$ Сибирский федеральный университет, Красноярск, Россия \\ ${ }^{2}$ Красноярский краевой Дом науки и техники РосСНИО, Красноярск, Россия \\ ${ }^{3}$ Сибирский научный центр ДНИТ, Красноярск, Россия \\ *E-mail: krasnio@bk.ru
}

\begin{abstract}
Аннотация. В статье представлен обзор основных направлений научной программы III Международного семинара MIP: Computing-2021: Компьютерное моделирование, информационные и вычислительные технологии (III International Workshop on Modeling, Information Processing and Computing - MIP: Computing-2021), который состоялся 28 мая 2021 в г. Красноярске на базе Красноярского краевого Дома науки и техники РосСНИО. Приведены обзорные сведения о работах, которые были включены в программу научной сессии семинара и представлены в виде онлайн / видео докладов и презентаций участников с размещением на сайте материалов семинара. Даны сведения о партнере семинара - сервисе научных публикации с открытым доступом CEUR-WS.org на Sun SITE Central Europe, работающем под эгидой технического университета в г. Аахене, Германия (RWTH Aachen University). Материалы семинара MIP: Computing-2021 в виде статей на английском языке опубликованы в CEUR Workshop Proceedings, том 2899.
\end{abstract}

Ключевые слова: информационные технологии, компьютерное моделирование, информатика, вычислительная техника

\section{1. Введение}

Третий Международный научный семинар по компьютерному моделированию, информационным и вычислительным технологиям (MIP: Computing-2021) прошел в Красноярске 28 мая 2021 года на базе Общественного учреждения «Красноярский краевой Дом науки и техники Российского Союза научных и инженерных общественных объединений» (ОУ «ККДНиТ») [1].

Семинар был совместно организован Международным и Российским союзом научных и инженерных общественных объединений (Москва, Россия), ОУ «ККДНиТ» и Сибирским научным центром ДНИТ (Красноярск, Россия). Бухарский государственный инженернотехнологический институт (Бухара, Узбекистан) выступил в качестве университета-партнера международного семинара. 
Modern Innovations, Systems and Technologies, 2021, 1(2)

Цель данного семинара заключалась в обмене опытом в области применения инноваций в информационных технологиях, современных методах математического моделирования и интеллектуальных системах как в теоретическом, так и прикладном аспекте, включая автоматизацию промышленного производства, методы защиты данных, образовательные технологии. К участию были приглашены ученые и специалисты российских и зарубежных вузов, академических институтов, предприятий, проектных и исследовательских центров. В семинаре приняли участие представители более 30 зарубежных и российских университетов, исследовательских центров.

Основные направления научной программы семинара:

- секция 1: «Кибернетика, вычислительное и имитационное моделирование»;

- секция 2: «Прикладная математика и бизнес-информатика»;

- секция 3: «Информационные технологии, надежность программного обеспечения и защита данных»;

- секция 4: «Обработка информации, разработка программного обеспечения и приложения».

На участие в семинаре было представлено 57 докладов. После рецензирования и рассмотрения материалов оргкомитетом MIP: Computing-2021 в сборник трудов семинара включены 28 статей, которые были распределены по двум следующим разделам:

- Кибернетика, вычислительные технологии и имитационное моделирование;

- Информационные технологии, надежность программного обеспечения и защита данных.

\section{2. Обзор докладов MIP: Computing-2021}

В данном обзоре представлены работы, которые были включены в программу пленарной сессии, в сборник трудов семинара MIP: Computing-2021 и представлены в виде онлайн/видео докладов и презентаций участников (размещены на сайте материалов семинара https://conf.domnit.ru/ru/materialy/mip-computing-2021/).

Работа [2] авторов А.А. Платонова, Н.А. Мамедовой на тему «Development of a methodology for cost optimization of software testing for the automatically tests generation» из Российского экономического университета имени Г.В. Плеханова (г. Москва) посвящена вопросам, связанным с процессом разработки и поддержки программного обеспечения (ПО), в частности, для тестирования программ. Решена задача разработки методики тестирования. Это позволяет оптимизировать затраты компании на автоматическую генерацию тестов. Проанализированы широко применяемые практики контроля качества программного обеспечения; сформулированы требования к разработанной методике. На основе 
разработанной методологии спроектирована ИТ-инфраструктура для контроля качества ПО. Новым результатом исследования являются инструменты для автоматической генерации качественных тестов, не требующих значительного преобразования архитектуры тестируемого программного обеспечения. Представленное решение может помочь компаниям существенно оптимизировать свои затраты на контроль качества разрабатываемого ПО. Объектом анализа и внедрения решений выступила компания - специализированный депозитарий, являющийся участником рынка ценных бумаг. Его информационная система представляет собой сложный многомодульный продукт. Для него реализована оригинальная практика повышения надежности ПО - покрытие кода тестами. Задача заключалась в обеспечении автоматической генерации тестов для поддержки бизнес-процессов специализированного депозитария.

В работе авторов из Брянского государственного технического университета Ивановой А.В., Сазоновой А.С., Кузьменко А.А., Филипповой Л.Б. на тему «Development and study of the model for epidemic spread» [3] рассматривается анализ и исследование эпидемиологической ситуации в Брянской области, вызванной вирусом COVID-19. Исследованы основные моменты разработки и тестирования имитационной модели распространения эпидемии. Предлагается алгоритм моделирования эпидемиологической ситуации, проводится оптимизационный эксперимент, который дает реалистичные значения на основе исторических данных, и делаются соответствующие выводы. Предлагаемый комплексный подход позволяет сократить финансовые средства и время, затрачиваемые на проведение экспериментов, а также позволяет прогнозировать динамику развития коронавируса в данном регионе для разработки стратегии оказания медицинских услуг с использованием информационных технологий, таких как методы системного моделирования.

В работе [4] авторов из Уфимского государственного нефтяного технического университета П. Кулакова, Б. Кутлубулатова, А. Рубцова, З. Мухамеджанова и В. Афанасенко на тему: «The assessment of impact of the crack size on the fracture load of a cylindrical element» рассматриваются вопросы одной из важнейших областей изучения материалов и конструкций - механика разрушения. Используется один из наиболее доступных в этой области инструментов - программа Ansys. Выполняемые в программном пакете расчеты на прочность конструкции, ее оценка по ресурсу и надежности во многих случаях проводятся с учетом возможного наличия в конструкции технологических или эксплуатационных трещин. Нарушение целостности конструкции может произойти по многим причинам: неправильный выбор материалов для изготовления конструкции, дефекты изготовления, превышение срока эксплуатации, экстремальные условия окружающей среды и т. д. С учетом перечисленного возникает необходимость в развитии отдельного направления исследований в данной области 
Modern Innovations, Systems and Technologies, 2021, 1(2)

знаний, создании оригинальных методов, посвященных анализу конструкций с трещинами различной природы по нескольким показателям. Это связано с тем, что ранее проектирование конструктивных элементов традиционно основывалось на результатах анализа прочности материала. Однако этот подход не подразумевает повышенного уровня напряжения из-за существующей в конструкции трещины, что может привести к неверным результатам.

Работа авторов из Санкт-Петербургского государственного университета аэрокосмического приборостроения и Санкт-Петербургского политехнического университета Петра Великого А. Туманова, В. Туманова, А. Титова и О. Узуна «Computer simulation of the safety of radio electronics production in an emergency situation» [5] посвящена компьютерному моделированию при решении задач обеспечения безопасности производственных процессов при воздействии поражающих факторов чрезвычайных ситуаций на примере организаций радиоэлектронной и приборостроительной промышленности. Организация любого производства связана с налаживанием ряда технологических процессов как основных, так и сопутствующих (обеспечивающих). Одним из важнейших сопутствующих процессов в радиоэлектронной и приборостроительной отраслях является процесс обеспечения безопасности и устойчивости производственной системы. В настоящее время эти процессы выходят на первый план, особенно когда становится актуальным налаживание производства в условиях прогнозируемых аварийных ситуаций. Выпускаемые изделия - радиоэлектронные устройства также должны соответствовать требованиям по термической, радиационной и механической стойкости. Если не учитывать факторы риска выхода из строя устройств и электронных компонентов, то даже небольшое отклонение параметров окружающей среды от нормальных значений приведет к техногенной аварии. Особенно это касается устройств, работающих в условиях ионизирующего излучения, больших перепадов температур и механических воздействий.

В работе [6] «Mathematical modeling of drive transitive process with linear change in control input» авторов Д. Ершова, И. Лукьяненко и Е. Злотникова из Санкт-Петербургского государственного университета аэрокосмического приборостроения и Санкт-Петербургского горного университета рассматриваются динамические параметры переходных процессов в электроприводе технологического оборудования в режиме разгона при изменении управляющего воздействия по линейному закону. Анализ динамической модели позволяет решать дифференциальные уравнения, описывающие три стадии в режиме разгона электропривода. Обсуждаются динамические и статические составляющие ошибок привода в зависимости от его скорости, обусловленной моментом нагрузки, общим моментом инерции привода, коэффициентом жесткости статического параметра и заранее заданным и запрограммированным ускорением. 
В работе авторов из Севастопольского государственного университета Д. Моисеева и А. Брюховецкого «Modeling the change detection process state of objects in monitoring data» [7] предложена модель обнаружения аномальных данных, основанная на исследовании однородности образцов. Метод предназначен для решения задачи обнаружения изменений в состоянии потока контролируемых данных с использованием моделей нормального и гаммараспределения на основе критерия непараметрической статистики Спирмена. Приведены результаты исследования значений интенсивности, влияющих на формирование запросов, интенсивность обслуживания приложений, загрузку системы, объем выборок, моменты времени измерения характеристик и уровни значимости изменений состояния объекта управления. Метод может использоваться для управления процессом обнаружения изменений в состояниях ресурсов UMV. В настоящее время процесс быстрого обнаружения аномалий в данных мониторинга критических объектов инфраструктуры является сложной, трудоемкой и трудно формализуемой задачей. Системы обнаружения вторжений являются наиболее эффективной мерой противодействия и наиболее надежным подходом к обеспечению защиты как традиционных, так и специализированных (например, автомобильных) компьютерных сетей.

Во второй работе этих же авторов «Method for detecting vulnerabilities of unmanned vehicle interfaces based on continuous values discretization» [8] предлагается подход, связанный c разработкой методов обеспечения безопасности беспилотных автомобилей в информационной инфраструктуре умного города. Метод основан на дискретизации непрерывных значений характеристик вектора состояния UMV-ресурсов, к которым относятся: канал связи, процессор, память. Для каждого из этих ресурсов предлагается оценить изменение таких характеристик, как степень загрузки ресурса и скорость ее изменения. Предлагаемый метод позволяет построить систему правил принадлежности анализируемых векторов указанным классам и минимизировать количество условий в сгенерированных правилах. Проблема обеспечения информационной безопасности беспилотных транспортных средств, работающих в интеллектуальных сетях транспортной инфраструктуры умного города, не теряет своей актуальности в связи с тем, что современные сети сталкиваются с беспрецедентным спектром компьютерных угроз, которые приводят к нарушению целостности, конфиденциальности и безопасности. доступность ресурсов.

В работе [9] авторов из МИРЭА - Российского технологического университета Т.Е. Смоленцевой, Е.Г. Бергер, И.С. Ганца «Description of the process of information flows formalization in the quality management system of education» рассматриваются информационные потоки, циркулирующие в системе управления качеством обучения (TQM) технических вузов при формулировании управляющих воздействий. Это позволяет детализировать 
информационные потоки, формализовать их, а также их взаимосвязь и получить схему с участием разработанной автоматизированной информационной системы поддержки принятия решений (АИС ППР). Проведен детальный анализ имеющейся информации в рассматриваемой системе, обеспечивающей добычу углеводородов. Это позволило определить наиболее полный набор параметров для первоначального развития АИС ППР. Для каждого элемента определены, в частности, цель, необходимая исходная информация, потребители полученной информации, представление данных и т.п.

Работа [10] авторов из Санкт-Петербургского государственного университета аэрокосмического приборостроения К. Епифанцева, О. Козловой «Research and development of artificial intelligence system for waste identification» рассматриваются возможности использования системы искусственного интеллекта для распознавания бытовых отходов. В статье сделан обзор современных программных продуктов для управления отходами. Авторы подчеркивают возможность разработки серии программных продуктов для управления отходами и развития компетенций в области управления отходами у жителей города СанктПетербурга. При решении поставленных задач используется программный продукт ЕСО-365, который также может быть полезен для улучшения экологии не только на региональном уровне. Представлена разработка еще одного программного продукта, позволившего с помощью искусственного интеллекта определять объемные (размерные) характеристики бытовых отходов. Акцент в данном исследовании сделан на программные продукты, используемые не только для распознавания пластика, но и на наиболее опасные с точки зрения экологии светодиодные лампы, аккумуляторы, в том числе литиевые блоки питания и батарейки. Отмечается, что создание представленных в работе фандоматов, увеличение их числа должно спровоцировать повсеместный рост экологического движения, популяризацию проектов раздельного сбора мусора.

В работе [11] авторов И. Сиддикова, Н. Мамасадыковой, О. Раимджановой, Д. Халматова, Х. Мирзаахмедова из Ташкентского государственного технического университета имени Ислама Каримова, Ташкентского университета информационных технологий имени Мухаммада аль-Хорезми и Ташкентского института текстильной и легкой промышленности рассматриваются вопросы синтеза нечеткой системы управления технологическими процессами. Представлен эффективный алгоритм синтеза контроллера нечеткой логики и нечеткой системы автоматического регулирования температурного режима химического реактора, инвариантного к параметрическим и внешним возмущениям. Предлагаемый алгоритм синтеза нечетко-логического пропорционально-интегрально-дифференциального (ПИД)-контроллера прост и позволяет использовать стандартную форму описания лингвистических переменных и минимальный набор правил управления. Синтезированный 
Современные инновации, системы и технологии, 2021, 1(2)

контроллер нечеткой логики дает возможность всей системе автоматического управления поддерживать температуру реактора на заданном уровне при наличии внешних возмущений, а также качественно управлять технологическим процессом с широким диапазоном изменения его параметров во времени. Используемые методы теории нечеткой логики и нейронных сетей позволяют оперировать лингвистическими нечеткими высказываниями. Сформированы основы правил логического вывода нечетко-логического регулятора в виде декартова произведения нечетких множеств с функцией принадлежности, имеющей трапециевидную форму. Результаты моделирования нечеткой системы управления показали, что при наличии в системе зашумленного внешнего сигнала и изменения его уровня до $30 \%$, а также изменения параметров объекта управления (усиление и постоянное время) до 25 \% (в сторону увеличения и уменьшения) нечеткая система сохраняет свойства устойчивости.

В работе [12] авторов из Ташкентского государственного технического университета, Ташкентского университета информационных технологий (ТУИТ) имени Мухаммада альХорезми, Ташкентского института текстильной и легкой промышленности и Самаркандского филиала ТУИТ И. Сиддикова, Н. Мамасадыковой, Д. Халматова, Н. Кадировой, О. Миржалилова, Г. Примовой «Development of neural network forecasting models of dynamic objects from observed data» рассматриваются вопросы построения прогнозных моделей состояния динамических моделей на основе нейросетевых структур, которые являются мощным математическим аппаратом для аппроксимации различных типов функций и оценки динамики изменения состояний рассматриваемых объектов. Предлагается метод построения прогнозных нейросетевых моделей для наблюдаемых данных реальных объектов с использованием гибридного приложения теории нелинейной динамики и нейросетей. Прогностическая модель нейронной сети, построенная на основе реконструированных фазовых траекторий процесса (аттрактора), позволит выбрать наиболее информативные характеристики, минимизировать архитектуру нейросети и размерность вектора на основе измеренных контролируемых сигналов. На базе разработанных алгоритмов предварительной обработки локальных участков реконструированного аттрактора и выбранного метода машинного обучения предложена схема построения нейросетевой прогнозной модели динамического поведения структурно сложной системы.

Работа [13] авторов из Ижевского государственного технического университета имени М.Т. Калашникова Д. Нефедова, К. Кетовой, И. Русяка на тему «Logistic planning for the use of renewable fuels in the energy complex of the region» посвящена решению проблемы логистического планирования при использовании возобновляемых видов топлива в энергетическом комплексе. Используемая технология - производство тепловой энергии из древесных отходов. Лесозаготовительные и деревообрабатывающие предприятия, 
Modern Innovations, Systems and Technologies, 2021, 1(2)

являющиеся источником древесных отходов, станции переработки древесных отходов, производящие щепу и топливные пеллеты, а также угольные котельные, вырабатывающие тепловую энергию, рассматриваются как объекты, участвующие в процессе производства тепловой энергии. Структура оптимального размещения участников процесса производства тепловой энергии из древесных отходов построена на примере Удмуртской Республики. В качестве оптимизации выбран экономический критерий минимизации затрат на производство тепла на всех угольных котельных региона с учетом затрат на поставку древесных отходов, а также на производство и поставку щепы и топливных пеллет. При численном решении применялся генетический алгоритм с реальным кодированием, а вычисления распараллеливались. Выяснилось, что в условиях Удмуртской Республики организация заводов по производству топливных пеллет оказалась нецелесообразной. Оптимальный вариант размещения предполагает наличие 6 точек для приготовления щепы. Это дает возможность обеспечить топливом все угольные котельные, расположенные в области. Средняя стоимость производства тепловой энергии на древесном топливе для всех рассматриваемых котельных составила 897 руб./Гкал, что на 76,6\% ниже соответствующего показателя для тепловой энергии, полученной из угля.

Работа [14] авторов из МИРЭА - Российского технологического университета Т.Е. Смоленцевой, А.В. Свищёва, Н.В. Катаховой «Designing an algorithm for supporting information generation process optimization» посвящена рассмотрению структурных элементов автоматизированной информационно-справочной системы поддержки принятия решений (AIRDSS) в иерархических многоуровневых сложных организационных системах. Сформулирована задача по обеспечению функционирования AIRDSS. Для ее решения были представлены основные этапы, которые включают вычисление коэффициентов важности для элементов вспомогательной информации (SI) и упорядочивание вариантов на основе предпочтений лица, принимающего решение, с последующим выбором перспективного по полезности варианта. На основе рассмотренной пошаговой схемы авторы предлагают алгоритм формирования оптимальной структуры элементов процедурного компонента AIRDSS для получения SI. Алгоритм имеет ряд преимуществ: простота расчета для различных экспериментов, относительно простая формализация экспертных знаний при представлении их числовыми значениями по степени важности.

Авторами из ИТМО и Санкт-Петербургского государственного университета аэрокосмического приборостроения А.В. Шукаловым, И.О. Жариновым и О.О. Жариновым представлено две работы в сборнике материалов семинара. В работе «Control of parallel computing in a cyber-physical production environment» [15] рассматриваются проблемы функциональной устойчивости производственного объекта Индустрии 4.0. Приведена 
структура киберфизической системы, компоненты которой управляются через специализированную сеть. Определены коммуникационные особенности программного управления оборудованием. Регулирование производственных процессов обеспечивается встроенной автоматизацией, поддерживающей инструменты онлайн-моделирования технологических процессов. Управление оборудованием выполняется с использованием оптимизационной модели, реализуемой на параллельных вычислительных системах. Коммуникационные механизмы управления кибер-объектами заимствованы из теории автоматических систем и теории сетей. Совместные расчеты позволяют управлять промышленным оборудованием по сети и настраивать коэффициенты регуляторов, отвечающих за корректное функционирование киберфизических систем. Повышение управляемости киберфизического производства основано на модификации потоков команд и данных, адаптивно изменяющихся под текущие технологические задачи. Многоцелевое управление реализуется через сеть, которая передает целевые регулирующие воздействия на контролируемые объекты. Наличие вычислительной, управляющей и сетевой составляющих обеспечивает функциональную стабильность процессов физической динамики производства, контролируемых программным обеспечением.

Вторая работа [16] «Network communication and identification of the cyber-physical systems» посвящена рассмотрению сетевых коммуникаций и идентификации киберфизических систем на основе элементов теории связи и теории информации, что позволяет определять динамические маршруты передачи данных. Коммутация сети выполняется с помощью системы концентраторов и маршрутизаторов для обеспечения распределенного подключения производственного оборудования на внутреннем и внешнем радиусе. Коммутация сети осуществляется при виртуальном построении системы подключения различных топологий для производственных процессов. Идентификация сети выполняется с помощью ряда физических, логических и информационных атрибутов, которые по своим значениям или свойствам четко соответствуют промышленным устройствам и системе коммутации. Показаны различные варианты адресации устройств и метаданные, которые важны для процедуры сетевой идентификации. Предложены варианты динамической топологии информационной сети, которые представляют операционную среду киберпроизводства с использованием принципов идентификации оборудования для маршрутизации и обработки технологических и управляющих данных.

В работе [17] авторов А.В. Гурьянова, А.Г. Коробейникова, И.О. Жаринова и О.О. Жаринова из Санкт-Петербургского ОКБ «Электроавтоматика», ИТМО (факультет безопасности информационных технологий), Санкт-Петербургского государственного университета аэрокосмического приборостроения, выполненной на тему «Edge, fog and cloud 
Modern Innovations, Systems and Technologies, 2021, 1(2)

computing in the cyber-physical systems networks» рассматривается параллелизм обработки данных при выполнении технологических задач, что является основой распределенной системы управления, которая используется в киберфизическом производстве (на основе облачных вычислений). Взаимное согласование рабочих и информационных производственных процессов обеспечивается технологией обмена сетевыми сообщениями, поддерживаемой функционально независимыми киберфизическими системами. Для управления очередью сообщений и координации действий промышленного оборудования используются элементы системы связи с функцией обработки потока данных в периферийных и облачных сетевых узлах и клиентских приложениях, размещенных в киберфизических устройствах системы. Собственные процессы управления промышленным оборудованием регулируются с помощью локальной вычислительной платформы. Управление в рамках обобщенной технологической задачи киберфизического производства обеспечивается многопроцессорной вычислительной платформой. Чтобы повысить качество обработки информации, фактически получаемой с помощью киберфизической системы, и ускорить обработку этих данных, выполняются облачные вычисления с задействованием ресурсов функциональных групп оборудования, разделенных в рамках общего информационного поля. Передача компонентов решения технологических задач на внешние элементы сети и реализация облачных вычислений высокой степени параллелизма в рамках производственной инфраструктуры - перспективный подход Индустрии 4.0 для организации взаимодействия современных производственных систем.

Работа [18] автора Д. Петрусевича «Improvement of time series forecasting quality by means of multiple models prediction averaging» из МИРЭА - Российского технологического университета посвящена построению моделей временных рядов. Обычно, требование простоты модели встроено в структуру информационных критериев, и наиболее подходящие модели не всегда являются лучшими с точки зрения значений критериев. Часто есть несколько лучших моделей, которые хорошо подходят для исследуемых временных рядов, и возникает проблема выбора между ними. Как правило, значения информационных критериев позволяют выбирать среди них. Но если нужна лучшая модель по качеству прогнозов, то исследователь может осуществить выбор и тестирование модели вручную. При этом, выбрав несколько моделей, можно построить их комбинацию. Самый простой способ - определить среднее значение их прогнозов и использовать его как комбинированный прогноз. Практические исследования подтверждают, что при таком подходе погрешность прогноза уменьшается. Также можно использовать более сложное построение, чем усреднение прогнозов (например, взвешенное голосование, которое широко используется при решении задач классификации). Но этот подход недостаточно теоретически обоснован. С теоретической точки зрения, 
построение доверительных интервалов прогнозов временных рядов также является достаточно сложной задачей. Интервалы, как правило, очень велики даже для моделей с хорошим качеством прогноза с точки зрения средних ошибок прогноза. Таким образом, интервалы прогнозирования используются редко. В данной статье построено несколько моделей временных рядов для индексов заработной платы и доходов (российских макроэкономических временных рядов). Их прогнозы объединяются в один прогноз, и его качество сравнивается с индивидуальными результатами прогнозов. Трансформация дисперсии прогноза и интервалов прогноза в случае простых (скользящие средние МА (q) и авторегрессии $\mathrm{AR}(\mathrm{p})$ низкого порядка) моделей также рассматривается, но это направление является частью дальнейшей работы.

В работе [19] автора К. Мухамадиевой из Бухарского инженерно-технологического института на тему «Fuzzy artificial neural network for prediction and management tasks» рассматривается задача прогнозирования параметров при построении систем прогнозирования и управления. Изучаются и анализируются существующие решения по использованию нечетких нейронных сетей. Предлагается структура полностью связанной нечеткой искусственной нейронной сети без слоя нечетких правил, соответствующая «классическому» многослойному персептрону. Различные структуры предлагаемой нечеткой искусственной нейронной сети протестированы на время обучения и среднеквадратичную ошибку при прогнозировании производительности угольной компании. Результаты позволяют выбрать количество нейронов в скрытых слоях в зависимости от желаемой точности предсказания выходного параметра.

В работе авторов Я.И. Шамлицкого, Д.В. Роговой, А.С. Поляковой, А.А. Попова и Л.В. Липинского «Models of analysis and forecasting of the traffic situation»[20] из Сибирского государственного университета науки и технологий имени академика М.Ф. Решетнева обсуждаются результаты анализа методов и моделей прогнозирования безопасности дорожного движения. Проблема создания математических моделей и программного обеспечения для описания и анализа транспортных процессов на сегодняшний день весьма актуальна. Исследованы и дан анализ дорожно-транспортных происшествий, установлены причинно-следственные связи дорожного движения и условий возникновения проблемных ситуаций. Решается задача разработки методологии прогнозирования и предотвращения дорожно-транспортных происшествий с целью снижения их количества. Приведен пример имитационной модели, описывающей маршрутную сеть города. Компьютерный эксперимент позволил отследить загруженность участков дорожной сети. Представленная работа направлена на повышение качества транспортной системы города за счет снижения вероятности аварий и исключения простоев маршрутных транспортных средств из-за заторов 
и пробок, а также способствует своевременной доставке пассажиров в заданное место назначения. С помощью разработанной модели можно изучить узкие места транспортных услуг, оценить дорожную обстановку, снизить напряженность и количество аварий на дорогах, улучшить экологическую обстановку и определить дальнейшие направления развития и совершенствования предложенного подхода.

Работа [21] авторов Д.Р. Калугина, Ю.А. Леонова, Р.А. Филиппова, Л.Б. Филипповой «Development of an information and analytical system for modeling the demographic situation in the Russian Federation» из Брянского государственного технического университета посвящена изучению показателей демографической статистики по субъектам Российской Федерации. В статье описывается актуальность данного исследования, а также преимущества и недостатки аналогичных программных решений: ИАС «Демография», витрины данных Росстата, автоматизированной ИС «Муниципальный регистр населения». Функциональная схема разработанной ИАС представлена как в виде единого функционального блока, так и в виде его декомпозиции на пять модулей, отвечающих за сбор аналитических данных, взаимодействие с базой данных, выявление корреляций, прогнозирование будущих показателей и визуализацию данных. На основании полученных данных можно проводить экстраполяцию, интерполяцию и регрессионный анализ данных. Авторы представили блок-схему алгоритма прогнозирования демографической статистики. Данный алгоритм используется для расчета прогноза демографической ситуации в Российской Федерации на основе информации, имеющейся в базе данных, с учетом пользовательских настроек. Рассмотрены функциональные возможности разработанной информационной системы. В качестве базы исследования авторы используют данные Федеральной службы государственной статистики (Росстат). Методы DataMining были использованы в качестве теоретической основы для разработки алгоритма прогнозирования. Разработанная информационная система предоставляет аналитику инструменты для анализа динамики, тенденций развития и определения взаимосвязи демографических показателей, а также построения прогноза их развития на ближайшие годы.

В работе [22] авторов из Самарского государственного технического университета А.В. Ненашева, А.Ю. Толстенко и Р.С. Олешко «Model of the peer-to-peer distributed system for securable information storage and processing without traffic prioritization (TheOoL project)» рассматривается математическая модель «Одноранговая распределенная система для защищенного хранения и обработки информации в корпоративных сетях». Это универсальная распределенная система, предназначенная для защиты распределенных вычислений и изоляции частных сетей без ограничения возможностей эффективного взаимодействия, криптографической безопасности, защиты от несанкционированного доступа с применением 
биометрии и инновационного протокола обмена данными для управления топологией на основе по технологии распределенного реестра. Моделирование проводилось с целью оценки производительности системы в зависимости от производительности аппаратного обеспечения ее узлов и телекоммуникационного оборудования сети.

В работе [23] авторов Амани Аль-Хусейн и В.Л. Стефанюк «The quantitative comparison between the integer splitting cipher and the traditional gamma cipher» из Российского университета дружбы народов, Института проблем передачи информации имени А. А. Харкевича РАН (ИППИ РАН) рассматривается шифр символьного целочисленного расщепления - это специальный математический метод, предложенный авторами, и его можно рассматривать как обобщение модульной арифметической операции. В этом шифре каждый текстовый символ представлен как целое число в соответствии с выбранной кодовой таблицей, после чего это целое число заменяется на основе другого числа последовательностью из $\mathrm{k}$ целых чисел (уровень k-разделения). Это исследование проводится с точки зрения возможной хакерской атаки. В статье доказаны две леммы, связанные с несанкционированным доступом к каналу передачи информации; первая лемма связана с вероятностным анализом несанкционированного восстановления открытого текста на основе гамма-шифра, а вторая лемма связана с вероятностным анализом несанкционированного восстановления открытого текста, обработанного криптосистемой с целочисленным разделением. После этого проводится количественное сравнение на основе этих двух лемм. В результате представленного исследования был сделан вывод о том, что эффективность расщепляющей криптосистемы существенно превосходит традиционный гамма-шифр, а также увеличение уровня расщепления приводит к большей защите информации и большему уровню безопасности.

Работа [24] авторов А. Брюховецкого, В. Миряновой, Д. Моисеева «Research of the model for detecting UMV interfaces vulnerabilities based on information criterion» из Севастопольского государственного университета рассматривает подход, связанный с разработкой методов обеспечения компьютерной безопасности беспилотных автомобилей (UMV). Подход основан на статистической оценке расстояния между распределениями вероятностей случайной величины. В качестве критерия оценки предлагается информационный критерий Дженсена-Шеннона, который обеспечивает симметричную версию дивергенции Кульбака-Лейблера. Обнаружение уязвимости выполняется на основе обработки значений состояния ресурсов UMV. Особенности мониторинга состояния UMV порождают новые проблемы, характеризующиеся потоками данных с переменной интенсивностью, неоднородными информационными потоками в условиях отсутствия априорной информации и зашумленных данных. При решении этой задачи возникают 
проблемы обработки больших данных: высокая вычислительная сложность из-за обработки больших объемов данных; высокая динамика контролируемых объектов; нестационарная информационная ситуация состояния объектов и окружающей среды; обеспечение высокой скорости обработки запросов; предоставление метрик информационных ресурсов в реальном времени.

В работе [25] авторов И.В. Ковалева, В.В. Лосева, М.В. Сарамуда, П.А. Кузнецова, А.С. Лифарь из Сибирского государственного университета науки и технологий имени академика М.Ф. Решетнева, Сибирского федерального университет, Красноярского государственного аграрного университета и China Aviation Industry General Aircraft Zhejiang Institute на тему «Formalization of the software module development using matrix forms» рассматривается формализованный подход к разработке многопользовательской системы обмена документами на основе матричной формы представления данных. В качестве базового процесса представлена многоэтапная разработка модульного программного обеспечения несколькими участниками. Представлена алгоритмическая реализация информационных потоков в контексте разработки программного обеспечения. Модель жизненного цикла документа описывается с помощью диаграмм UML. Рассматриваемый алгоритм содержит иерархическую структуру документооборота с подчинением одних документов другим. В соответствии с документооборотом рассматривается модель взаимодействия участников процесса разработки программного обеспечения. Модель административного документооборота включает в себя следующие роли: менеджер по разработке продукта; представитель заказчика; разработчик; тестер. Представленные матрицы полностью описывают график рассматриваемого документооборота. Таким образом, формализованный подход, основанный на матричной форме представления, может быть использован в качестве входных данных для алгоритмизации многопользовательских систем при решении задач модульного проектирования программного обеспечения.

Работа [26] авторов Д. Моисеева и В. Миряновой «Models of the threat of virus idea dissemination in information-telecommunication networks» из Севастопольского государственного университета посвящена решению проблем, связанных с распространением вирусов. Рассматривается область исследования процессов реинтеграции постконфликтных обществ. Как известно, информационные и телекоммуникационные сети в настоящее время включают в себя всевозможные средства переключения абонентов, наиболее распространенными и популярными являются социальные сети, которые по сути предоставляют практически полный набор возможностей для обмена мультимедийной информацией между пользователями. Очевидно, что многие информационные сети «мгновенно воспринимаются активными участниками социальных сетей». Эффективная 
защита абонентов от угрозы распространения идеи вируса - серьезная проблема, особенно для развития и реинтеграции постконфликтных обществ, поскольку современный Интернет дает не только мобилизационные и технологические возможности, но и оказывает информационное и психологическое воздействие на индивидуальное и массовое сознание. В свою очередь, идею вируса следует понимать как некое информационное сообщение, которое часто «подбрасывается» СМИ и моментально улавливается активной частью подписчиков социальных сетей.

Работа [27] авторов из Сибирского федерального университета, Сибирского государственного университета науки и технологий имени академика М.Ф. Решетнева С. Зиннер, В. Иваненко, В. Тынченко, П. Волегжанина, А. Сташкевича «Using machine learning methods in problems with large amounts of data» исследует использование искусственного интеллекта в медицине, в частности в радиологии, разработке лекарственных средств. Раскрыта полезность роботов-помощников в медицине. Рассмотрены вопросы машинного обучения в медицине, а также задачи маршрутизации в больницах. Также обсуждаются такие методы машинного обучения, как методы классификации, методы восстановления регрессии, методы кластеризации. Делается вывод о том, что ручная обработка становится более сложной и невозможна при больших объемах данных. Возникает потребность в автоматической обработке, которая может трансформировать современную медицину. Авторами сделаны выводы о том, насколько точно механизмы глубокого обучения могут обеспечить более точный результат обработки и классификации радиологических изображений по сравнению с результатами, полученными медицинскими специалистами и экспертами. Стало ясно, что глубокое обучение не только помогает в выборе и извлечении характеристик радиологических изображений, но также имеет потенциал для измерения прогнозных целевых аудиторий и предоставления упреждающих прогнозов, чтобы помочь клиницистам ускорить их повседневную деятельность.

В работе [28] авторов Б. Шакирова, Л. Хайруллиной, Л. Мингайлиевой, В. Бронской, Г. Хабибуллиной, Е. Фадеевой, О. Харитоновой «Using emotional evaluation of text in a foreign language learning app» из Казанского федерального университета и Казанского национального исследовательского технологического университета рассматривается современный уровень развития компьютерной лингвистики, который характеризуется вовлечением все более сложных уровней лингвистического анализа в область автоматического анализа, использованием гибридных подходов к решению задач компьютерной обработки текстов, сочетающих машинное обучение и алгоритмические методы. В то же время уровни сложности современных задач обработки текста, такие как извлечение временной привязки в тексте, анализ структуры дискурса и многие другие, требуют активного привлечения экспертных 
лингвистических знаний. В настоящее время изучение иностранного языка является целью очень большого количества людей. Существует множество инструментов, методов и приемов саморазвития при освоении иностранного языка, что позволяет значительно повысить эффективность обучения. В настоящее время сентимент-анализ используется в системах мониторинга, аналитики и сигнализации, а также в системах документооборота и рекламы. При этом эта технология практически не используется в системах обучения иностранным языкам, хотя имеет огромный нереализованный потенциал. Практически все существующие на данный момент приложения для изучения иностранных языков не используют возможность анализа тональности текста. Овладение эмоциональной лексикой языка - сложная задача для ученика, и этому аспекту следует уделять особое внимание. Представленная статья посвящена описанию разработанного авторами веб-приложения для изучения немецкого языка, которое основано на использовании метода анализа тональности речи. Данное решение можно распространить и на другие иностранные языки.

Работа С. Васильева «Classification of methods of moving the scanning sensor of a mechatronic profiler along the trajectories of plane curves» [29] из Чувашского государственного университета имени И.Н. Ульянова и Нижегородского государственного университета рассматривает актуальные вопросы использования анализа плоских кривых для классификации способов перемещения сканирующего датчика мехатронного профилометра. Основная цель исследования - определение возможности классификации способов перемещения сканирующего датчика мехатронного профилометра по траекториям плоских кривых по характеристикам, полученным при анализе этих плоских кривых. Согласно спиральной кривой Архимеда, большое количество сгустков точек будет сосредоточено в центральной части исследуемой области, что в целом делает исследование весьма трудоемким. Для детального изучения поверхности необходимо задать оптимальную траекторию движения датчика профилометра по рассчитанной плоской кривой. Выяснилось, что использование траектории движения датчика по спирали Ферма более перспективно, так как количество сканируемых точек в центре меньше и будет пропорционально количеству точек на элементарных участках, равномерно распределенных на исследуемой площади. Классификация способов перемещения сканирующего датчика мехатронного профилометра произведена на основе результатов, полученных при исследовании траекторий плоских кривых.

\section{3. Заключение}

Статьи по материалам докладов, представленных на семинаре, соответствуют тематике научной программы, отражают новые научно-практические результаты, полученные авторами 
в области информационных технологий, математического моделирования и интеллектуальных систем.

Все докладчики пленарной сессии и авторы видео-докладов, представленных на семинаре, отмечены дипломами оргкомитета MIP: Computing-2021. Участники, представившие Е-презентации по материалам докладов с размещением их на сайте семинара, получили сертификаты об участии в работе MIP: Computing-2021.

Партнером семинара выступил центральноевропейский сервис научных публикации с открытым доступом CEUR-WS.org на Sun SITE Central Europe, работающий под эгидой технического университета в г. Аахене, Германия (RWTH Aachen University). Материалы семинара MIP: Computing-2021 в виде статей на английском языке опубликованы в CEUR Workshop Proceedings, том 2899 [1-29].

\section{Список литературы}

[1] Kovalev, I. III International workshop on modeling, information processing and computing (MIP: Computing-2021): Preface. / I. Kovalev, E. Semenkin // CEUR Workshop Proceedings. - 2021. - № 2899. - P. 0-1.

[2] Platonov, A.A. Development of a methodology for cost optimization of software testing for the automatically tests generation / A.A. Platonov, N.A. Mamedova // CEUR Workshop Proceedings. - 2021. - № 2899. - P. 1-8. https://doi.org/10.47813/dnit-mip3/2021-2899-18.

[3] Ivanova, A. Development and study of the model for epidemic spread. / A. Ivanova, A. Sazonova, A. Kuzmenko, L. Filippova // CEUR Workshop Proceedings. - 2021. - № 2899. - P 9-16. https://doi.org/10.47813/dnit-mip3/2021-2899-9-16.

[4] Kulakov, P. The assessment of impact of the crack size on the fracture load of a cylindrical element / P. Kulakov, B. Kutlubulatov, A. Rubtsov, Z. Mukhametzyanov, V. Afanasenko // CEUR Workshop Proceedings. - 2021. - № 2899. - P. 17-24. https://doi.org/10.47813/dnit$\operatorname{mip} 3 / 2021-2899-17-24$.

[5] Tumanov, A. Computer simulation of the safety or radio electronics production an emergency situation / A. Tumanov, V. Tumanov, A. Titov, O. Uzun // CEUR Workshop Proceedings. - 2021. - № 2899. - P. 25-30. https://doi.org/10.47813/dnit-mip3/2021-2899-25-30.

[6] Ershov, D. Mathematical modeling of drive transitive process with linear change in control input / D. Ershov, I. Lukjanenko, E. Zlotnikov // CEUR Workshop Proceedings. - 2021. № 2899. - P. 31-35. https://doi.org/10.47813/dnit-mip3/2021-2899-31-35.

[7] Byukhovetskiy, A. Modeling the change detection process state of objects in monitoring data / A. Byukhovetskiy, D. Moiseev // CEUR Workshop Proceedings. - 2021. - № 2899. - P. 36-42. https://doi.org/10.47813/dnit-mip3/2021-2899-36-42. 
[8] Moiseev, D. Method for detecting vulnerabilities of unmanned vehicle interfaces based on continuous values discretization / D. Moiseev, A. Bryukhovetskiy // CEUR Workshop Proceedings. - 2021. - № 2899. - P. 43-47. https://doi.org/10.47813/dnit-mip3/2021-289943-47.

[9] Smolentseva, T. E. Description of the process of information flows formalization in the quality management system of education / T. E. Smolentseva, E.G. Berger, I.S. Gantz // CEUR Workshop Proceedings. - 2021. - № 2899. - P. 48-53. https://doi.org/10.47813/dnitmip3/2021-2899-48-53.

[10] Epifantsev, K., Research and development of artifical intelligence system for waste indification / K. Epifantsev, O. Kozlova // CEUR Workshop Proceedings. - 2021. - № 2899. - P. 5463. https://doi.org/10.47813/dnit-mip3/2021-2899-54-63.

[11] Siddikov, I. Algorithms for synthesis of a fuzzy control system chemical reactor temperature / I. Siddikov, N. Mamasodikova, O. Rayimdjanova, D. Khalmatov, X. Mirzaaxmedova // CEUR Workshop Proceedings. - 2021. - № 2899. - P. 64-70. https://doi.org/10.47813/dnitmip3/2021-2899-64-70.

[12] Siddikov, I. Development of neural network forecasting models of dynamic objects from observed data / I. Siddikov, N. Mamasodikova, D. Khalmatov, N. Kadirova, O. Mirjalilov, G. Primova // CEUR Workshop Proceedings. - 2021. - № 2899. - P. 71-77. https://doi.org/10.47813/dnit-mip3/2021-2899-71-77.

[13] Nefedov, D. Logistic planning for the use of renewable fuels in the energy complex of the region / D. Nefedov, K. Ketova, I. Rusyak // CEUR Workshop Proceedings. - 2021. - № 2899. - P. 78-84. https://doi.org/10.47813/dnit-mip3/2021-2899-78-84.

[14] Smolentseva, T.E. Designing an algorithm for supporting information generation process optimization / T.E. Smolentseva, A.V. Svishchev, N.V. Katakhova // CEUR Workshop Proceedings. - 2021. - № 2899. - P. 85-92. https://doi.org/10.47813/dnit-mip3/2021-2899$85-92$.

[15] Shukalov, A.V. Control of parallel computing in a cyber-physical production environment / A.V. Shukalov, I.O. Zharinov, O.O. Zharinov // CEUR Workshop Proceedings. - 2021. - № 2899. - P. 93-97. https://doi.org/10.47813/dnit-mip3/2021-2899-93-97.

[16] Shukalov, A.V. Network communication and identification of the cyber-physical systems / A.V. Shukalov, I.O. Zharinov, O.O. Zharinov // CEUR Workshop Proceedings. - 2021. - № 2899. - P. 98-102. https://doi.org/10.47813/dnit-mip3/2021-2899-98-102.

[17] Gurjanov, A.V. Edge, fog and cloud computing in the cyber-physical systems networks / A.V. Gurjanov, A.G. Korobeynikov, I.O. Zharinov, O.O. Zharinov // CEUR Workshop Proceedings. - 2021. - № 2899. - P. 103-108. https://doi.org/10.47813/dnit-mip3/2021- 
2899-103-108.

[18] Petrusevich, D.A. Improvement of time series forecasting quality by means of multiple models prediction averaging / D.A. Petrusevich // CEUR Workshop Proceedings. - 2021 . - № 2899. - P. 109-117. https://doi.org/10.47813/dnit-mip3/2021-2899-109-117.

[19] Mukhamadieva, K. Fuzzy artificial neural network for prediction and management tasks / K. Mukhamadieva // CEUR Workshop Proceedings. - 2021. - № 2899. - P. 118-124. https://doi.org/10.47813/dnit-mip3/2021-2899-118-124.

[20] Shamlitskiy, Y. Models of analysis and forecasting of the traffic situation / Y. Shamlitskiy, D. Rogova, A. Polyakova, A. Popov, L. Lipinskiy // CEUR Workshop Proceedings. - 2021. № 2899. - Pp 125-132. https://doi.org/10.47813/dnit-mip3/2021-2899-125-132.

[21] Kalugin, D. Development of an information and analytical system for modeling the demographic situation in the Russian Federation / D. Kalugin, Y. Leonov, R. Filippov, L. Filippova // CEUR Workshop Proceedings. - 2021. - № 2899. - P. 133-140. https://doi.org/10.47813/dnit-mip3/2021-2899-133-140.

[22] Nenashev, A.V. Model of the peer-to-peer distributed system for securable information storage and processing without traffic prioritization (THEOOL project) / A.V. Nenashev, A.Y. Tolstenko, R.S. Oleshko // CEUR Workshop Proceedings. - 2021. - № 2899. - P. 141-150. https://doi.org/10.47813/dnit-mip3/2021-2899-141-150.

[23] Alhussain, A. The quantitative comparison between the integer splitting cipher and the traditional gamma cipher / A. Alhussain, V. Stefanuk // CEUR Workshop Proceedings. 2021. - № 2899. - P. 151-161. https://doi.org/10.47813/dnit-mip3/2021-2899-151-161.

[24] Bryukhovetskiy, A. Research of the model for detecting UMV interfaces vulnerabilities based on information criterion / A. Bryukhovetskiy, V. Miryanova, D. Moiseev // CEUR Workshop Proceedings. - 2021. - № 2899. - P. 162-168. https://doi.org/10.47813/dnit$\operatorname{mip} 3 / 2021-2899-162-168$.

[25] Kovalev, I.V. Formalization of the software module development using matrix forms / I.V. Kovalev, V.V. Losev, M.V. Saramud, P.A. Kuznetsov, A.S. Lifar // CEUR Workshop Proceedings. - 2021. - № 2899. - P. 169-174. https://doi.org/10.47813/dnit-mip3/20212899-169-174.

[26] Moiseev, D. Models of the threat of virus idea dissemination in information-telecommunication networks / D. Moiseev, V Miryanova // CEUR Workshop Proceedings. - 2021. - № 2899. - P. 175-180. https://doi.org/10.47813/dnit-mip3/2021-2899-175-180.

[27] Zinner, S. Using machine learning methods in problems with large amounts of data / S. Zinner, V. Ivanenko, V. Tynchenko, P. Volegzhanin, A. Stashkevich // CEUR Workshop Proceedings. - 2021. - 2899. - P. 181-187. https://doi.org/10.47813/dnit-mip3/2021-2899- 
Modern Innovations, Systems and Technologies, 2021, 1(2)

181-187.

[28] Shakirov, B. Using emotional evaluation of text in a foreign language learning app / B. Shakirov, L. Khairullina, L. Mingalieva, V. Bronskaya, G. Khabibullina, E. Fadeeva, O. Kharitonova // CEUR Workshop Proceedings. - 2021. - № 2899. - P. 188-195. https://doi.org/10.47813/dnit-mip3/2021-2899-188-195.

[29] Vasiliev, S. Classification of methods of moving the scanning sensor of a mechatronic profiler along the trajectories of plane curves / S. Vasiliev // CEUR Workshop Proceedings. - 2021. - № 2899. - P. 196-202. https://doi.org/10.47813/dnit-mip3/2021-2899-196-202. 